\title{
Optimal Design of Adaptive Laminated Beam Using Layerwise Finite Element
}

\begin{abstract}
Abolghassem Zabihollah and Shahin Zareie
Sharif University of Technology, International Campus, Kish Island, Iran

Correspondence should be addressed to Abolghassem Zabihollah, zabihollah@sharif.edu

Received 30 September 2010; Revised 30 January 2011; Accepted 21 February 2011

Academic Editor: Bernhard Jakoby

Copyright ( $) 2011$ A. Zabihollah and S. Zareie. This is an open access article distributed under the Creative Commons Attribution License, which permits unrestricted use, distribution, and reproduction in any medium, provided the original work is properly cited.

First, an efficient and accurate finite element model for smart composite beams is presented. The developed model is based on layerwise theory and includes the electromechanical coupling effects. Then, an efficient design optimization algorithm is developed which combines the layerwise finite element analysis model for the smart laminated beam, sensitivity analysis based on analytical gradients and sequential quadratic programming (SQP). Optimal size/location of sensors/actuators is determined for dynamic displacement measurement purposes and for vibration control applications. For static and eigenvalue problems, the objective is to minimize the mass of the beam under various constraints including interlaminar stresses, displacements, and frequencies. For transient vibration problems, the objective is the minimization of the actuation control effort to suppress the vibration in a controlled manner. Illustrative examples are provided to validate the formulation and to demonstrate the capabilities of the present methodology.
\end{abstract}

\section{Introduction}

Laminated composite structures with sensing and actuation capabilities so called as smart laminated structures potentially have numerous applications in modern industries including aerospace and automotive industries. Piezoelectric materials are the most appropriate sensing/actuating elements utilized in smart laminated structures. These novel smart structures combine the superior mechanical properties of conventional composite materials and incorporate the additional inherent capability of piezoelectric layers to sense and adapt their static and dynamic response.

Due to the presence of large number of material and geometrical parameters as well as loading conditions, the determination of the optimal design of the system becomes a crucial issue in smart laminated structures. The conventional design methodology leads to very long and expensive procedures which sometimes make the design infeasible, thus requiring a robust design optimization algorithm. Design optimization of smart isotropic structures has been tackled for variety of purposes and objectives such as the determination of optimal location of actuators/sensors for vibration control [1-3], optimal sensor location for vibration measurements [4], and optimal location of actuator for shape control applications [5-7]. However, design optimization of smart laminated structures still is in the preliminary level, and many issues need to be explored, including structural modeling and functions gradients, in order to come up with a robust optimization algorithm.

Structural modeling of the smart system plays an essential role in the efficiency and accuracy of the design optimization algorithm. Most of the structural models of smart laminated structures have been developed based on the equivalent single-layer (ESL) theories $[8,9]$ in which the displacement field is assumed to be continuous through the thickness. This assumption results in continuous transverse strain through the thickness. However, the stiffnesses of the adjacent layers are not equal, thus requiring discontinuity in transverse stress through the thickness which is in contrary to the equilibrium of the interlaminar forces. In general, the single-layer theories provide acceptable results for relatively thin laminates. For thick laminates and for laminates with high inhomogeneities through the thickness, the ESL theories lead to erroneous results [10]. It has been shown that 
the presence of interlaminar stresses near the free edges of laminated structures, including smart laminated structures, is significant which may not accurately be determined by ESL theories [11]. Apparently, exceeding the interlaminar stresses between the host laminated structure and bonded/embedded sensor/actuator (particularly at the free edges) may break the bonding between the smart elements and the host structure, thus it results in the loss of the main functionality, namely, sensing and actuating capability of the smart laminate. Therefore, monitoring the interlaminar stresses in this novel structure is an essential issue for any design methodology.

Application of different materials (sensor, actuator, composite, etc.) and geometries (thickness, size, and location) to construct a smart laminated structure causes strong inhomogeneities through the thickness. Therefore, a robust electromechanical model is required to account for the material and electromechanical inhomogeneities in these hybrid laminates and to provide accurate prediction of the active response of the structures.

Robbins and Reddy [12] improved the analysis of smart laminated structures by proposing a layerwise displacement formulation to investigate the static and dynamic interaction between a piezoelectric actuator and an underlying laminated beam. The layerwise formulation has the capability to address the local through-the-thickness effect, such as the evolution of complicated stress-strain fields in smart composite beams and the interfacial phenomena between the embedded microdevises and passive composite plies in a smart laminate. Recently, layerwise theory has been applied for the analysis of laminated beam with piezoelectric actuators for shape control [13] and for free vibration [14].

Most of available works in the field of design optimization of smart laminated structures are developed based on the structural modeling using classical laminate and 3 firstorder shear deformation theories [15-17]. Yan and Yam [18] considered the bending moment induced by piezoelectric patch actuators as objective function to investigate the optimal thickness and embedded depth of piezoelectric patches in active vibration control. In an attempt to improve the accuracy of the design optimization algorithm, Carlos et al. [19] presented a finite element model based on the higherorder shear deformation theory to determine the optimal design of laminated composite plate structures. The objective of optimization in this work was the determination of the optimum locations of the piezoelectric actuators in order to maximize their efficiency for static deformation control. Chee et al. [20] used a higher-order shear deformation theory for displacement field and a layerwise approximation for electric field to determine the optimal actuator layout for shape control applications.

Another dominant factor in any gradient-based design optimization procedures is the evaluation of functions gradients which computationally influence the accuracy and efficiency of the procedure. A coupled layerwise finite element model has been recently developed by the authors [21] for sensitivity analysis of smart composite beams in which the analytical functions gradients for various purposes are presented. In the present work, the layerwise theory for structural modeling along with the corresponding analytical gradients are combined with sequential quadratic programming (SQP) technique to develop an efficient design optimization algorithm for smart laminated beams.

Optimal size/location of sensors/actuators is determined for dynamic displacement measurement purposes and for vibration control applications. For static and eigenvalue problems, the objective is to minimize the mass of the beam under various constraints including interlaminar stresses, displacements, and frequencies. For transient vibration problems, the objective is the minimization of the actuation control effort to suppress the vibration in a controlled manner. Illustrative examples are given to validate the formulation and to demonstrate the capabilities of the present model.

1.1. Mathematical Modeling. The displacement and electric potential fields in a laminated beam based on the layerwise theory are given by

$$
\begin{aligned}
& u(x, z, t)=\sum_{l=1}^{N} U_{l}(x, t) \Phi(z), \\
& w(x, z, t)=\sum_{l=1}^{N} W_{l}(x, t) \Phi(z), \\
& \psi(x, z, t)=\sum_{l=1}^{N} \psi_{l} \Phi(z),
\end{aligned}
$$

where $u$ and $w$ are displacements along $x$ and $z$-directions, respectively, $\psi$ is the electrical potential, $N$ is the total number of nodes through the thickness, $\Phi(Z)$ is the $1 \mathrm{D}$ Lagrange interpolation function along the $z$-direction which is defined between any two adjacent layers. For thin laminate, displacements in $z$-direction between layers are negligible, so $w(x, z, t)=W_{0}(x, t)$.

Finite element formulation of the smart laminated beam has been obtained by incorporating local in-plane approximations for the state variables introduced in (1) as follows:

$$
\begin{aligned}
U_{l} & =\sum_{i=1}^{N_{n}} U_{l}^{i}(x) \varphi(x), \\
W_{l} & =\sum_{i=1}^{N_{n}} W_{l}^{i}(x) \varphi(x), \\
\psi & =\sum_{i=1}^{N_{n}} \psi_{l}^{i}(x) \varphi(x),
\end{aligned}
$$

where $N_{n}$ is the number of nodes, and $\varphi_{i}(x)$ are the interpolation functions along the length of the beam, respectively. In practical applications, the bottom and upper surfaces of piezoelectric patches are covered with conductive materials requiring constant electrical potential at the covered surfaces; thus, $\psi_{I}(x, t)=\psi_{I}^{o}$. By implementing the in-plane approximations for the state variables into the variational form of the state equation of motion and collecting the coefficients of displacements, $\{U\},\{W\}$, and $\{\psi\}$, the following matrix 
equation for the smart laminated beam can be obtained as follows:

$$
\begin{aligned}
& {\left[\begin{array}{cc}
{\left[M_{u u}\right]} & {[0]} \\
{[0]} & {[0]}
\end{array}\right]\left[\begin{array}{l}
\{\ddot{u}\} \\
\{\ddot{\psi}\}
\end{array}\right]+\left[\begin{array}{ll}
{\left[k_{u u}\right]} & {\left[k_{u \psi}\right]} \\
{\left[k_{\psi u}\right]} & {\left[k_{\psi \psi}\right]}
\end{array}\right]\left[\begin{array}{l}
\{u\} \\
\{\psi\}
\end{array}\right]} \\
& \quad=\left[\begin{array}{l}
\{F(t)\} \\
\{Q(t)\}
\end{array}\right],
\end{aligned}
$$

where $\left[k_{u u}\right],\left[k_{\psi}\right]$, and $\left[k_{\psi \psi}\right]$ stand, respectively, for the elastic, piezoelectric, and permittivity matrices. Further, $\left[M_{u u}\right]$ is the mass matrix, and $\{F(t)\}$ and $\{Q(t)\}$ are the applied mechanical load vector and electrical charge vector, respectively. The displacement $\{u\}$ represents a vector containing axial and transverse displacements as $\{u\}=$ $U W^{T}$ represents the electric potential.

Considering the electric potential vector as $\{\psi\}=$ $\left\{\psi^{s}, \psi^{a}\right\}$, where $\left\{\psi^{s}\right\}$ and $\psi^{a}$ represent, respectively, the voltage output at the sensor and the voltage imposed on the actuator layer, (3) can be expressed in a compact form with the electric potential partitioned into active and sensory components as

$$
\begin{aligned}
& {\left[\begin{array}{rr}
{\left[M_{u u}\right]} & {[0]} \\
{[0]} & {[0]}
\end{array}\right]\left[\begin{array}{c}
\{\ddot{u}\} \\
\{\ddot{\psi}\}
\end{array}\right]+\left[\begin{array}{cc}
{\left[k_{u u}\right]} & {\left[k_{u \psi}^{s s}\right]} \\
{\left[k_{\psi u}^{s s}\right]} & {\left[k_{\psi \psi}^{s s}\right]}
\end{array}\right]\left[\begin{array}{l}
\left\{u^{s}\right\} \\
\{\psi\}
\end{array}\right]} \\
& =\left[\begin{array}{c}
\left\{F(t)-\left[k_{u \psi}^{s a}\right]\left\{\psi^{a}\right\}\right\} \\
\left\{Q^{s}(t)-\left[k_{\psi \psi}^{s a}\right]\left\{\psi^{a}\right\}\right\}
\end{array}\right],
\end{aligned}
$$

where superscripts $s$ and $a$ stand for partitioned submatrices in accordance with the sensory and actuator components, respectively. In the left hand side, $\{u\}$ and $\left\{\psi^{s}\right\}$ indicate the nodal displacements and voltage at the sensors, respectively. In the right hand side, $\left\{F_{u}\right\},\left\{\psi^{a}\right\}$, and $\left\{Q^{s}\right\}$ are, respectively, the known applied mechanical load, applied voltage at the actuator, and the electric charge at the sensors. Assuming the electric charge at sensors $\left\{Q^{s}\right\}$ to be zero, (4) can also be condensed into the following independent equations to determine the generated voltage at the sensor as

$$
\left\{\psi^{s}\right\}=-\left[K_{\psi \psi}^{s s}\right]^{-1}\left(\left[K_{\psi u}^{s s}\right]\{u\}+\left[K_{\psi \psi}^{s a}\left\{\psi^{a}\right\}\right]\right),
$$

and the structural displacements as

$$
\begin{aligned}
& {\left[M_{d d}\right]\{\ddot{u}\}+[\hat{K}]\{u\}} \\
& \quad=\left\{F_{u}(t)\right\}\left(\left[k_{\psi \psi}^{s s}\right]\left[k_{\psi \psi}^{s s}\right]^{-1}\left[k_{\psi a}^{s a}\right]-\left[k_{u \psi}^{s s}\right]\right)\left\{\psi^{a}\right\},
\end{aligned}
$$

where $[\hat{K}]=\left(\left[K_{u u}\right]-\left[K_{\psi u}^{s s}\right]\left[K_{\psi \psi}^{s s}\right]^{-1} K_{u \psi}^{s s}\right)\left\{\psi^{a}\right\}$.

\section{Static Problem}

Static problems in smart laminated structures usually referred to problems dealing with controlling the shape due to applied external load where the system basically works in actuating mode. For static problems, (6) is reduced to

$$
[\hat{K}]\{u\}=\{F\},
$$

where the external loading consists of mechanical load, $\left\{F_{u}(t)\right\}$, and electric loading due to applied voltage at actuator, $\left\{F_{P}(t)\right\}$, and given by

$$
\{F\}=\left\{F_{p}(t)\right\}+\left\{F_{u}(t)\right\},
$$

where

$$
\left\{F_{p}(t)=\left(\left[K_{u u}\right]-\left[K_{\psi u}^{s s}\right]\left[K_{\psi \psi}^{s s}\right]^{-1} K_{u \psi}^{s s}\right)\left\{\psi^{a}\right\}\right\} .
$$

In actuator mode and in the absence of the sensors in the structure, the piezoelectric load is reduced to $\left\{F_{p}(t)\right\}=$ $-\left(K_{u \psi}^{s a}\right)\left\{\psi^{a}\right\}$.

\section{Dynamic Problem}

Dynamic problems in smart laminated structures typically referred to the problems concerning with vibration control and measurements. For dynamic problems considering structural damping, (6) may be written as

$$
\left[M_{u u}\right]\{\ddot{u}\}+[C]\{\dot{u}\}+[\hat{K}]\{u\}=\left\{F_{u}(t)+F_{p}(t)\right\},
$$

where matrix $[C]$ is the structural damping matrix of the system.

\section{Design Optimization}

Sequential quadratic programming (SQP) is a widely used method for most complex nonlinear optimization problems owing to its robustness and high efficiency in searching for the optimum point. Considering a general problem of minimizing the objective function $f(q)$, subject to constraint $g(q) \leq 0$, we can write the Lagrangian function as

$$
L(\{q\}, \lambda)=\sum_{i=1}^{m} \lambda_{i} \cdot g_{i}(\{q\})+F(\{q\}),
$$

where $\lambda_{i}$ is the Lagrangian multiplier. Then, the quadratic programming $(\mathrm{QP})$ subproblem can be formulated based on a quadratic approximation of the Lagrangian function as

$$
\min \frac{1}{2} d^{T} H_{k} d+\delta f\left(q_{k}\right)^{T} d
$$

subject to

$$
\begin{aligned}
& \nabla g_{i}\left(q_{k}\right)^{T} d+g_{i}\left(q_{k}\right)=0, \\
& \nabla g_{i}\left(q_{k}\right)^{T} d+g_{i}\left(q_{k}\right) \leq 0,
\end{aligned}
$$

where $\{q\}, g_{i}$, and $\lambda_{i}$ and $i$ are design variable vector, constraint functions, and Lagrange multipliers, respectively.

The matrix $H_{k}$ is a positive definite approximation of the Hessian matrix of the Lagrangian function and can be updated by using quasi-Newton methods. The solution to the subproblem is then used to form a new iteration until convergence occurs.

To improve the chances of obtaining a minimum closer to global minimum, a trial and error or heuristic approach 
TABLE 1: Material properties used in numerical examples.

\begin{tabular}{lccc}
\hline Properties & NCT/301 & Adhesive & PZT \\
\hline$E_{1}(\mathrm{Gpa})$ & 144.3 & 6.9 & 63.0 \\
$E_{3}(\mathrm{Gpa})$ & 9.85 & 6.9 & 63.0 \\
$\nu_{13}$ & 0.28 & .4 & 0.28 \\
$G_{13}(\mathrm{Gpa})$ & 4.34 & 2.46 & 24.8 \\
$d_{31}(\mathrm{~m} / \mathrm{V})$ & - & - & $-166 e(-12)$ \\
$g_{33}(\mathrm{farad} / \mathrm{m})$ & - & - & $11.53 e(-9)$ \\
$\rho\left(\mathrm{kg} / \mathrm{m}^{3}\right)$ & 1385 & 1662 & 7600 \\
\hline
\end{tabular}

is used together with the SQP method. A simple heuristic method involves randomly selecting a set of starting points in the hope that one of these starting points is close to the global minimum. While global minimum is not assured, the probability of obtaining better minimum increases with the number of starting points. For more details of the SQP procedure, one may consult the book written by Arora [22]. Examining (12) and (13), one can easily realize that the gradients of objective and constraint functions $f\left(q_{k}\right)$ and $g_{i}\left(q_{k}\right)$ are the most dominant factor in convergence of the optimization procedure. As it is mentioned above, in order to reach the global optimum, the procedure should be repeated for different starting points, requiring an efficient computations of the function gradients.

In the present work, the analytical gradient of objective and constraint functions have been derived based on the layerwise finite element formulation [21] and implemented in SQP method for all the optimization problems unless otherwise specified.

\section{Numerical Solution}

This section presents the results for several representative problems. The effects of various design variables on the design objective and constraints are also investigated. All the applications focus on a laminated cantilever beam with embedded or surface-bonded piezoelectric sensors and/or actuators. The properties of the material used for all the example applications can be found in Table 1.

5.1. Mass Minimization. Laminated structures integrated with piezoceramic elements are widely used in aerospace applications where the mass of the structures is a major concern. However, the density of piezoceramic materials is roughly four times higher than graphite/epoxy material (commonly used composite material for aerospace applications); thus, minimizing the mass while maintaining the functionality of the system is one of the main issues in designing smart laminated structures. In this section mass minimization of smart laminated beam is studied for different design constraints, including deflection, interlaminar stresses, and natural frequencies.

5.1.1. Deflection Constraint. In this problem, the optimal thicknesses of both piezoelectric sensor and actuator patches have been determined in order to achieve the minimum mass

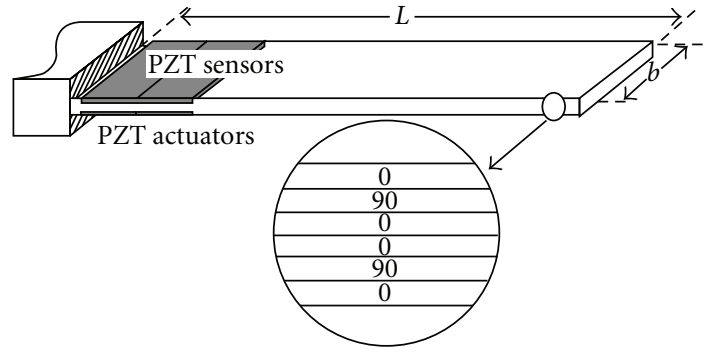

FIgURE 1: Schematic illustration of piezo-laminated cantilever beam.

of the beam. The beam consists of six layers of $0.125 \mathrm{~mm}$ thick graphite/epoxy and is configured as $[0 / 90 / 0]_{s}$. The length and width of the beam are $L=0.3 \mathrm{~m}$ and $b=0.03 \mathrm{~m}$, and it is meshed with 10 equal-length elements in which the two $\left(3 \times 3 \mathrm{~cm}^{2}\right)$ piezoelectric patches are mounted at the first two elements on top surface as sensing device, and another two similar patches are mounted at the bottom surface of the laminate as actuating devices as shown in Figure 1. PZT actuators are excited by $100 \mathrm{~V}$ electric potential.

The objective of this problem is to obtain the thicknesses of both sensors and actuators to minimize the mass of the beam where the tip deflection, tip $u_{u p}$, is greater than $0.1 \mathrm{~mm}$ and the generated voltage at sensors, $\psi^{s} s$, is considered to be higher than 6 volts to acquire a strong enough sensor voltage. The optimization problem can be cast into the following form:

$$
f=\sum_{j=1}^{N_{\mathrm{PZT}}} b \rho_{i}^{\mathrm{PZT}} t_{j}^{\mathrm{PZT}}+\sum_{i=1}^{N_{\mathrm{PZT}}} b \rho^{\mathrm{PZT}} t_{j}^{\mathrm{PZT}} \longrightarrow \min ,
$$

subject to $u_{u p} \geq 0.1 \mathrm{~mm}, \psi^{s} \geq 6 \mathrm{~V}$.

Considering the constant thickness for composite layers, the second term in (14) becomes a constant value of $1.558 \mathrm{gr}$, where $\rho^{\mathrm{PZT}}, t^{\mathrm{PZT}}$, and $N_{\mathrm{PZT}}$ denote the material density, thickness, and number of PZT patches, respectively; similarly, $\rho^{c}$, $t^{c}$, and $N$ stand for the material density, layer thickness, and number of composite layers, respectively. The optimization problem is solved by implementing the layerwise finite element formulation in sequential quadratic programming (SQP), and the optimal thickness 8 for both sensor and actuator is found to be $0.5 \mathrm{~mm}$ which results in $0.118 \mathrm{~mm}$ tip deflection, 5.20 -volt sensor voltage, and the minimum mass of $11.3 \mathrm{gr}$,

$$
f=\sum_{j=1}^{N_{\mathrm{PZT}}} b \rho_{i}^{\mathrm{PZT}} t_{j}^{\mathrm{PZT}}+\sum_{i=1}^{N_{\mathrm{PZT}}} b \rho^{\mathrm{PZT}} t_{j}^{\mathrm{PZT}} \longrightarrow \min ,
$$

subject to

$$
\begin{gathered}
u_{u p}=0.2 \mathrm{~mm}, \\
\left(\tau_{x z}\right) \leq 1000 \mathrm{MPa}, \\
1.0 \mathrm{~V} \leq \psi^{a} \leq 100 \mathrm{~V}, \\
0.1 \mathrm{~mm} \leq t^{c} \leq 20.0 \mathrm{~mm}, \\
0.1 \mathrm{~mm} \leq t^{\mathrm{PZT}} \leq 20.0 \mathrm{~mm} .
\end{gathered}
$$


TABLE 2: Optimal layer thickness ( $\mathrm{mm}$ ) and PZT voltage (volt).

\begin{tabular}{lcccccc}
\hline \multirow{2}{*}{ Laminate } & \multicolumn{6}{c}{ Layer thickness } \\
& $L 1$ & $L 2$ & $L 3$ & $L 4$ & $L 5$ & $L 6$ \\
\hline$[0 / 90]_{s}$ & 0.1 & 0.1 & 0.888 & 0.889 & 0.889 & 0.887 \\
{$[ \pm 45]_{s}$} & 0.1 & 0.1 & 0.913 & 1.110 & 0.963 & 0.8973 \\
{$[ \pm 30]_{s}$} & 0.1 & 0.1 & 0.1 & 0.810 & 0.790 & 0.887 \\
\hline
\end{tabular}

TABle 3: PZT thickness (mm) and voltage (volt).

\begin{tabular}{lccc}
\hline Laminate & Top PZT $(\mathrm{mm})$ & Bottom PZT $(\mathrm{mm})$ & Voltage $(\mathrm{V})$ \\
\hline$[0 / 90]_{s}$ & .1 & .887 & 72.2 \\
{$[ \pm 45]_{s}$} & .1 & .887 & 66.33 \\
{$[ \pm 30]_{s}$} & .1 & .887 & 91.5 \\
\hline
\end{tabular}

The thicknesses of composite and PZT layers as well as the applied voltage are determined for laminate configurations, $[0 / 90]_{3 T},[ \pm 45]_{3 T}$, and $[ \pm 30]_{3 T}$. The results are provided in Tables 2 and 3 in which one can realize that configuration $[ \pm 45]_{3 T}$ requires higher layers thickness and lower actuating voltage than that of the $[0 / 90]_{3 T}$ and $[ \pm 30]_{3 T}$. This is due to the lowest stiffness of configuration $[ \pm 45]_{3 T}$. Consequently, configuration $[ \pm 30]_{3 T}$ needs lower layer thickness and higher actuating voltage than that of $[0 / 90]_{3 T}$.

Iteration procedure leading to the optimal mass for different laminate configurations is provided in Figure 2.

As it was expected from the conclusion derived from the results provided in Tables 2 and 3, the minimum mass is obtained, and configurations are in the second and third ranks. The interlaminar stresses corresponding to the optimal design are presented in Figure 3. It is indicated that interlaminar stresses between the laminate layers and piezoelectric layers are higher than the interlaminar stresses between host layers. This is due to the higher value of shear stiffness for piezoelectric materials.

This result is of great importance in designing smart laminated beam since the force applied by the PZT actuators usually is very small. In terms of manufacturing, the bonding between piezoelectric elements and host structure should be well treated in order to prevent detaching of actuators from the composite layers

$$
f=\sum_{j=1}^{N_{\mathrm{PZT}}} b \rho_{i}^{\mathrm{PZT}} t_{j}^{\mathrm{PZT}}+\sum_{i=1}^{N_{\mathrm{PZT}}} b \rho^{\mathrm{PZT}} t_{j}^{\mathrm{PZT}} \longrightarrow \min .
$$

In addition, the applied voltage should be kept in a safe region for PZT ceramics. It is observed that the optimal thickness of the upper and lower PZT patches greatly depends on the lamination orientation and applied voltage. It should be noted that monitoring interlaminar stresses in optimization procedure when using ESL theories is not straightforward and also it is not accurate. It requires indirect and not-so-accurate computations, and also it is very time consuming. However,the finite element model based

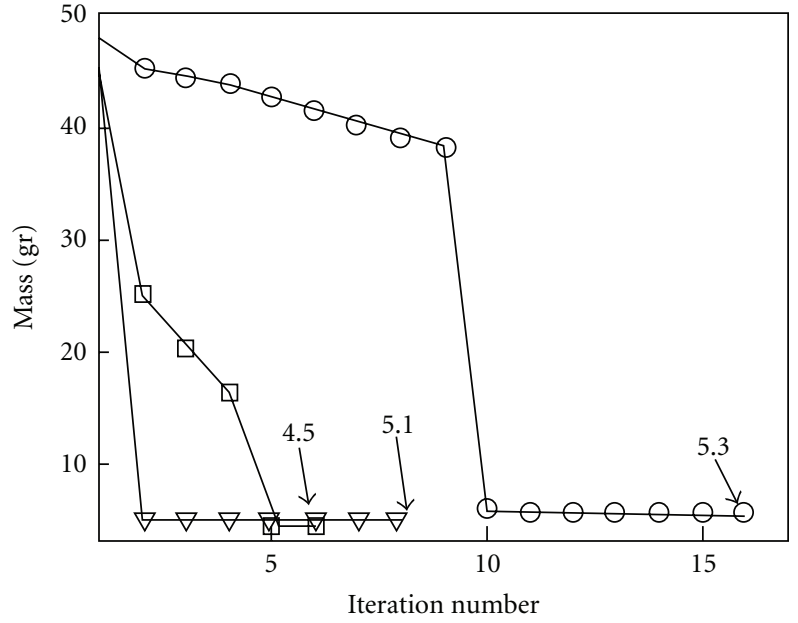

$$
\begin{aligned}
& -( \pm 45)_{3 T} \\
& \square( \pm 30)_{3 T} \\
& \nabla(0 / 90)_{3 T}
\end{aligned}
$$

FIGURE 2: Iteration history to minimize the mass.

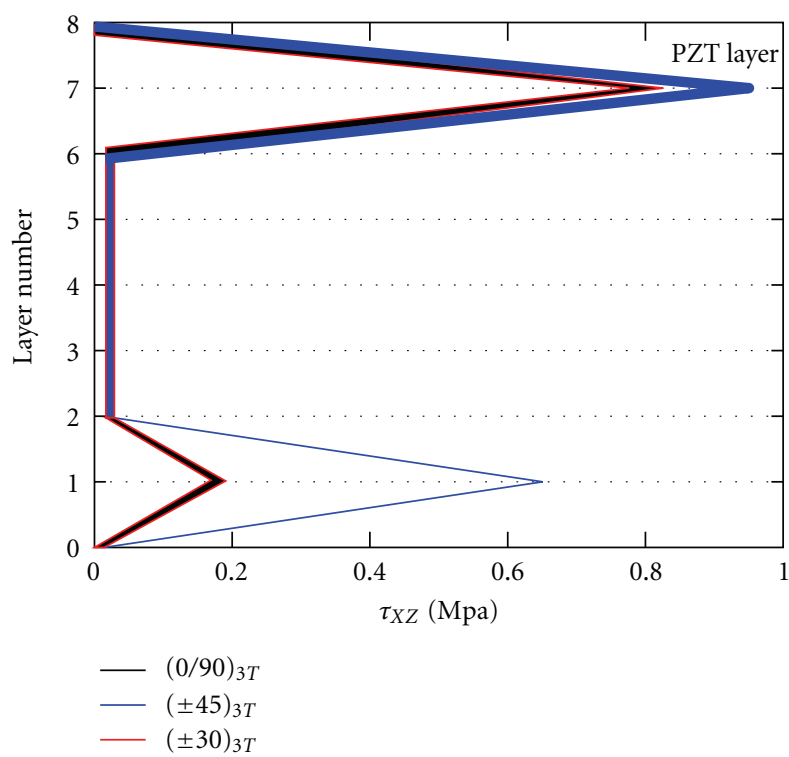

Figure 3: Optimal interlaminar stresses.

on layerwise theory provides interlaminar stresses that are very close to the results obtained using three-dimensional elements [10]. Therefore, layerwise theory provides an efficient (in terms of computation) and accurate (compared to $3 \mathrm{D}$ analysis) tool in the design optimization procedure.

5.1.2. Natural Frequency Constraint. The smart laminated beam described in Section 5.1.1 is again considered here to determine the optimal thickness of composite layers to minimize the mass while the thickness of both sensors and actuators is assumed to be $0.5 \mathrm{~mm}$. The first three natural frequencies of the smart composite beam are monitored not to exceed 40,215 , and $680 \mathrm{~Hz}$, respectively. The optimization 
TABLE 4: Optimal layer thickness with frequency constraint.

\begin{tabular}{lccc}
\hline & Layer thickness & Total mass & Reduces mass \\
\hline Initial design & $1.0(\mathrm{~mm})$ & $82.0(\mathrm{gr})$ & 0.0 (percent) \\
Optimal (no coupling) & $0.59(\mathrm{~mm})$ & $58.55(\mathrm{gr})$ & 28.65 (percent) \\
Optimal (coupling) & $0.54(\mathrm{~mm})$ & $56.0(\mathrm{gr})$ & 34.14 (percent) \\
\hline
\end{tabular}

problem is written in formal constraint optimization format as

$$
f=\sum_{j=1}^{N_{\mathrm{PZT}}} b \rho_{i}^{\mathrm{PZT}} t_{j}^{\mathrm{PZT}}+\sum_{i=1}^{N_{\mathrm{PZT}}} b \rho^{\mathrm{PZT}} t_{j}^{\mathrm{PZT}} \longrightarrow \min ,
$$

subject to $\left(\omega_{1}, \omega_{2}, \omega_{3}\right) \leq(40,250,680)$.

Considering the constant mass of the PZT elements, the first term is determined as 27.36 gr. The optimal design for layer thickness is obtained considering with and without electromechanical coupling effect in the finite element model and given in Table 4. It is observed that performing optimization technique may relatively reduce the total mass of the beam by 28 percent when neglecting the effect of electromechanical coupling in the analysis procedure. Considering electromechanical effect in the finite element model may decrease the mass by 34 percent.

\section{Actuator Force Minimization}

6.1. Damping Constraint. A cantilever laminated beam with 4 layers [0/90/90/0] each of $0.125 \mathrm{~mm}$ thickness is considered. The length and width of the beam are given as $0.33 \mathrm{~m}$ and $0.0254 \mathrm{~m}$, respectively. One piezoelectric actuator $(16.5 \times$ $25.8 \mathrm{~mm}^{3}$ ) is attached on the top surface at $0.05 \mathrm{~m}$ from the fixed end of the beam. The free end of the beam is initially displaced by $10 \mathrm{~mm}$. It is desired to determine the minimum electric potential to suppress the transient vibration in a controlled manner. It is required to reduce the settling time to $0.5 \mathrm{sec}$ by applying electric potential to the actuators. The optimization problem is minimization of the control effort in time interval 0 to $T$. The problem is cast into the following standard format:

$$
f=\int_{0}^{T} V^{2}(t) d t \longrightarrow \min
$$

subject to $W(t)=0,|V(t) \leq v(a)|$, settling time $=0.5 \mathrm{sec}$, where $T$ is the allowable time to suppress the motion and is assumed to be $2 \mathrm{sec}, V_{a}$ is the maximum applied voltage on the actuators (1000 volt), and $w$ represents the transverse displacement of the beam.

Figure 4 shows the initial response of the laminated beam and the response of the system after the optimum voltages given in Figure 5 are applied to the system. It is observed that by performing the optimization, the settling time is reduced from 2.5 seconds to $0.5 \mathrm{sec}$. In Figure 6 , the iteration history of the performance function (control effort) is shown. In the first iteration, the control effort is jumped to its maximum level, and after six iterations, it converged to its minimum value which is about $10000 \mathrm{~V}^{2} \cdot \mathrm{sec}$. Initial and optimal control forces are presented in Figure 7, where

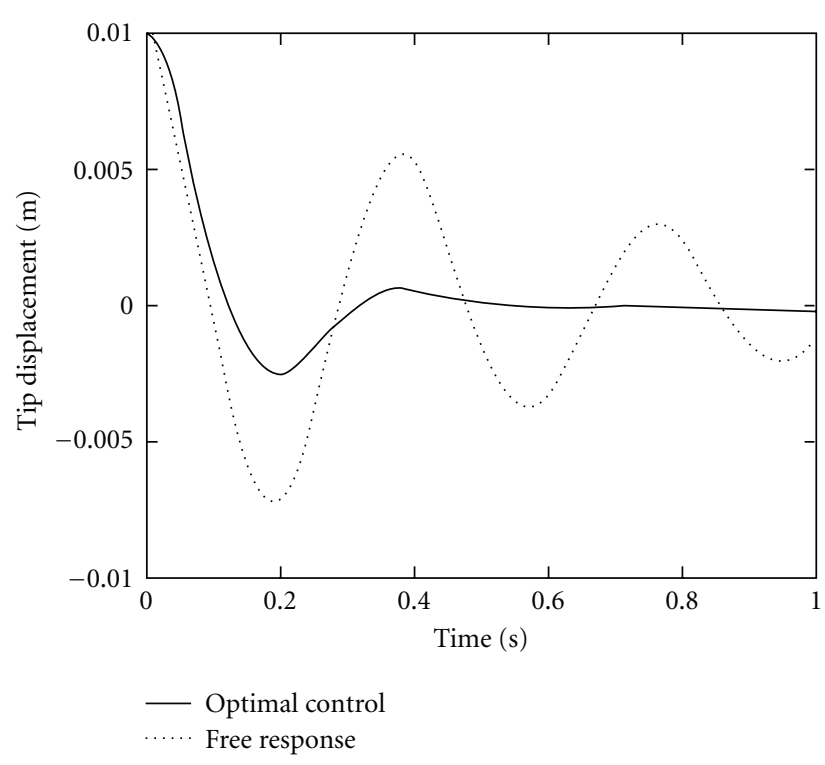

FIGURE 4: Vibration suppression using optimal electric potential.

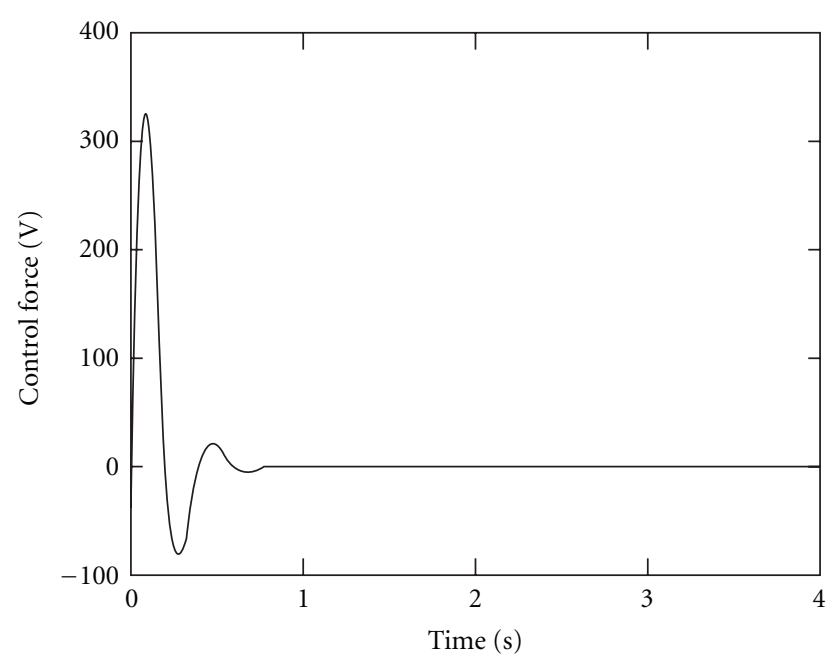

FIgURE 5: Optimal voltages applied at actuators.

one may realize a significant reduction in the first peak. Obviously, the difference is a relative value, and it depends on the initial design.

6.2. Damping Constraint. Further, the effects of laminate configuration and location of actuator are investigated. The smart laminated beam of the previous example is considered except that the laminate configuration and location of the actuator are changed for different cases.

In the first case, the actuator, $A$, is attached at the upper surface $([A / 0 / 90 / 90 / 0])$. For the second case, the actuator is embedded inside the laminate, namely $([0 / 90 / A / 90 / 0])$. In the third case, the effect of laminate configuration is studied by making a smart laminated beam as ([A/45/-45/-45/45]).

In Figures 8 and 9, the optimal tip response and the optimal control force of the laminated beam with different configurations are presented. 


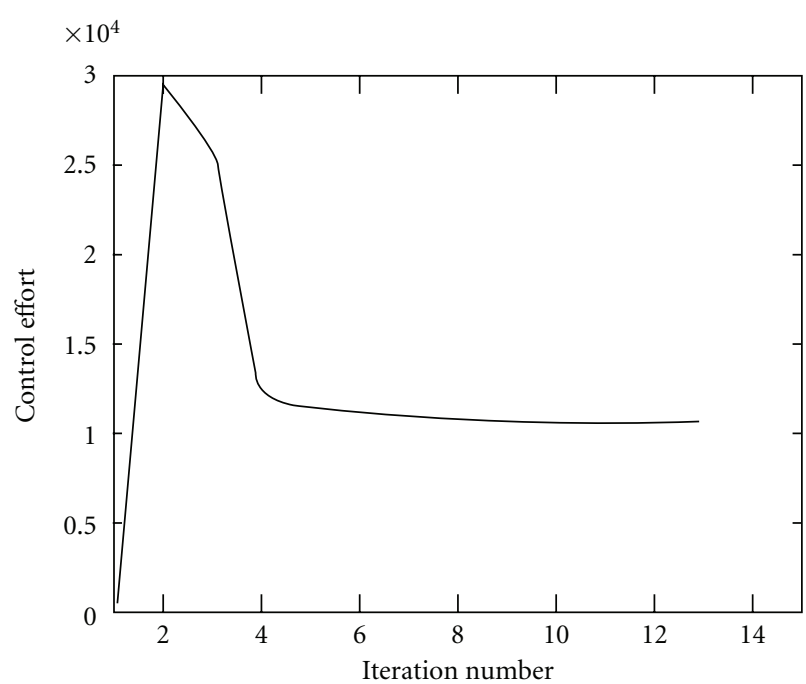

FIGURE 6: Performance history for the optimal control problem.

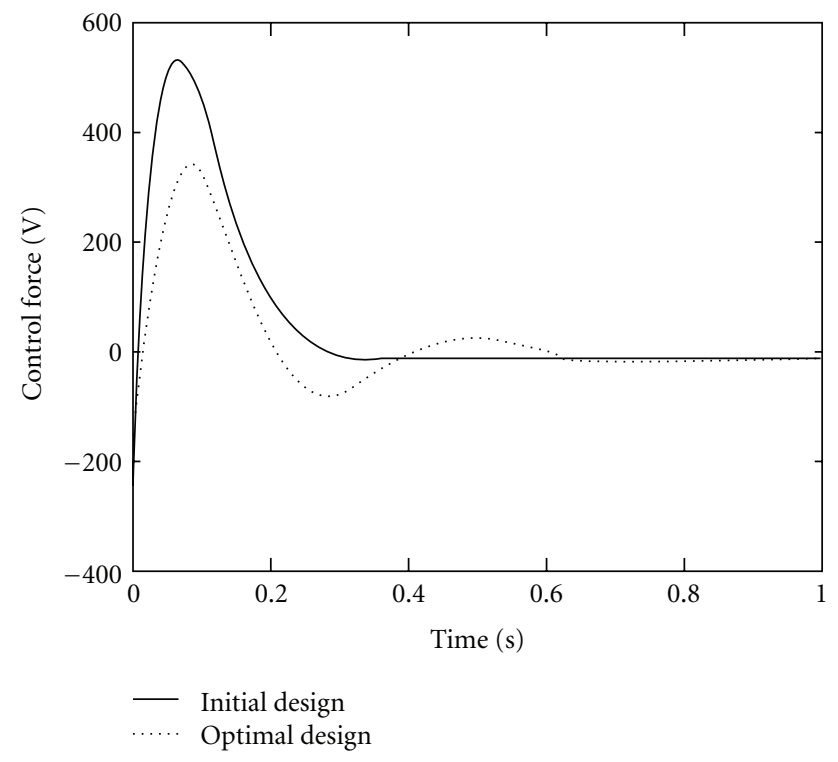

FIGURE 7: Initial and optimal control forces control problem.

As it is observed from Figure 4, the settling time for all the cases is $0.5 \mathrm{sec}$ as it was determined in optimization problem. Figure 9 indicates that the laminated beam configured as $[A / 45 /-45 /-45 / 45]$ requires higher value of control force in order to suppress the vibration in $0.5 \mathrm{sec}$. This value is slightly reduced for the laminate configuration $[0 / 90 / A / 90 / 0]$ which is due to the change in structural stiffness of the beam. The value of control effort and the objective of the optimization problem for $[A / 45 /-45 /-45 / 45],[0 / 90 / A / 90 / 0]$, and $[A / 0 / 90 / 90 / 0]$ are determined as $75.8 \times 10^{4}, 58 \times 10^{4}$ and $10.1 \times 10^{4}$, respectively. One may realize that the lower structural stiffness of configuration $[A / 45 /-45 /-45 / 45]$ leads to higher vibration amplitude; thus, it requires higher control effort to suppress the vibration response. On the other hand, relocation of actuator elements from outer surface to inner surface increases the required control effort

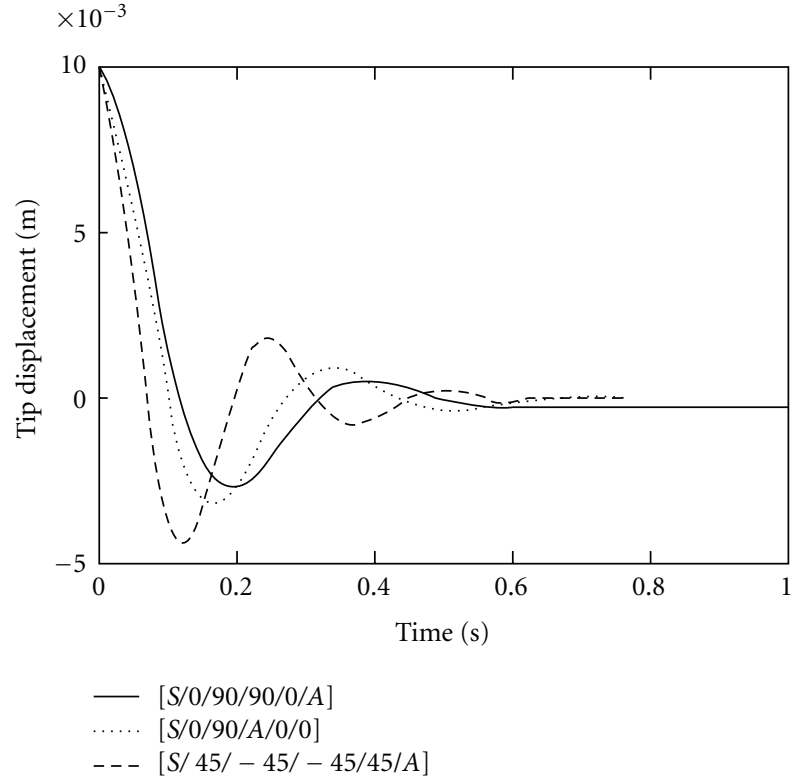

FIGURE 8: Effects of laminate configuration and location of actuator on vibration suppression using optimal electric potential.

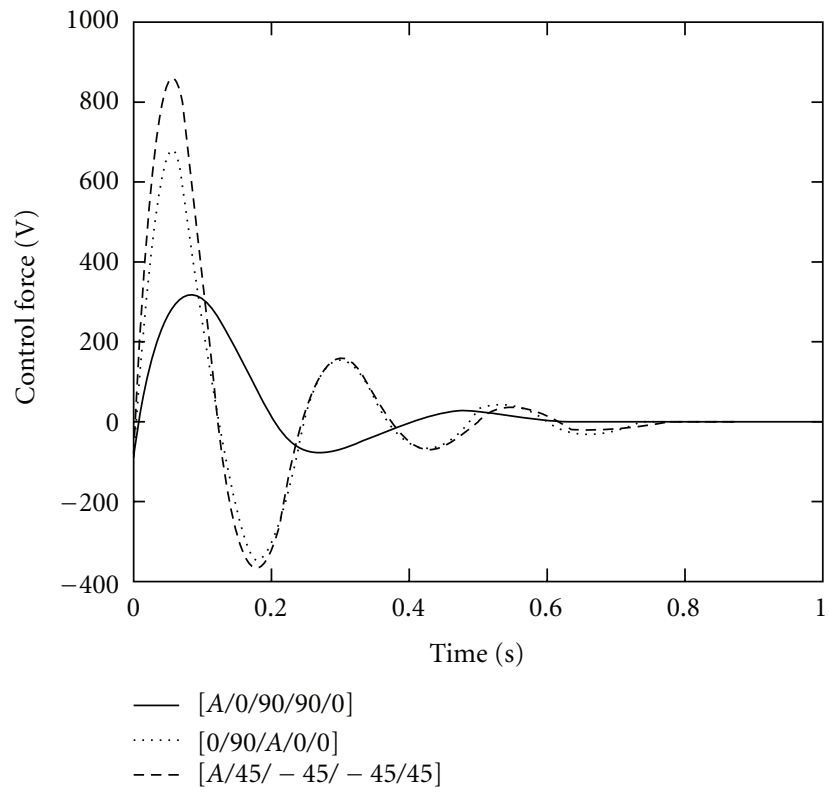

FIGURE 9: Effects of laminate configuration and location of actuator on optimal voltages applied at actuators.

which is due to the higher moment effect of outer actuating element compared to the inner ones.

\section{Conclusion}

Smart laminated composite beams with surface-bonded/ embedded piezoelectric layers as sensors and/or actuators have been investigated based on the layerwise displacement theory. A finite element formulation with considering electromechanical effects has been developed to investigate 
the effects of the size and location of piezoelectric patches on the design parameters. The developed finite element formulation for the coupled analysis of smart laminated beams along with analytical function gradients have been combined with sequential quadratic programming to develop an efficient design optimization algorithm. Utilizing the developed algorithm, the optimal design of smart laminated beams for various constraints and objectives has been investigated. In static case, nodal displacement and interlaminar stresses are considered as constraints to minimize the mass. In dynamic problems, natural frequencies are monitored as design constraints for eigenvalue problem, and the minimization of control effort is considered for transient vibration. Due to the higher accuracy of displacement and stresses obtained from layerwise theory, the optimal designs of smart laminated structures based on the layerwise approach are more reliable than that of the equivalent single layer theories. In addition, utilizing analytical gradients in sequential quadratic programming leads to faster convergence to the optimal design.

\section{Acknowledgment}

The authors wish to acknowledge the support provided by Sharif University of Technology, International Campus, and Kish Water and Power Company on Kish Island.

\section{References}

[1] I. Bruant, G. Coffignal, F. Lene, and M. Verge, "A methodology for determination of piezoelectric actuator and sensor location on beam structures," Journal of Sound and Vibration, vol. 243, no. 5, pp. 861-882, 2001.

[2] H. Zheng, G. S. H. Pau, and Y. Y. Wang, "A comparative study on optimization of constrained layer damping treatment for structural vibration control," Thin-Walled Structures, vol. 44, no. 8, pp. 886-896, 2006.

[3] J. Osama, "Optimal size and location of piezoelectric actuator/sensors: practical considerations," Journal of Guidance, Control, and Dynamics, vol. 23, no. 3, pp. 509-514, 2000.

[4] Z. N. Li, J. Tang, and Q. S. Li, "Optimal sensor locations for structural vibration measurements," Applied Acoustics, vol. 65, no. 8, pp. 807-818, 2004.

[5] E. P. Hadjigeorgiou, G. E. Stavroulakis, and C. V. Massalas, "Shape control and damage identification of beams using piezoelectric actuation and genetic optimization," International Journal of Engineering Science, vol. 44, no. 7, pp. 409421, 2006.

[6] S. Adali, J. C. Bruch, I. S. Sadek, and J. M. Sloss, "Robust shape control of beams with load uncertainties by optimally placed piezo actuators," Structural and Multidisciplinary Optimization, vol. 19, no. 4, pp. 274-281, 2000.

[7] R. Barboni, A. Mannini, E. Fantini, and P. Gaudenzi, "Optimal placement of PZT actuators for the control of beam dynamics," Smart Materials and Structures, vol. 9, no. 1, pp. 110-120, 2000.

[8] J. N. Reddy, "On laminated composite plates with integrated sensors and actuators," Engineering Structures, vol. 21, no. 7, pp. 568-593, 1999.
[9] A. Suleman and V. B. Venkayya, "Simple finite element formulation for a laminated composite plate with piezoelectric layers," Journal of Intelligent Material Systems and Structures, vol. 33, pp. 775-786, 1995.

[10] J. Reddy, Mechanics of Laminated Composite Plates, Theory and Analysis, CRC Press, New York, NY, USA, 1997.

[11] M. Tahani and A. Nosier, "Edge effects of uniformly loaded cross-ply composite laminates," Materials and Design, vol. 24, no. 8, pp. 647-658, 2003.

[12] D. Robbins and J. Reddy, "Analysis of piezoelectrically actuated beams using a layerwise displacementtheory," Composite and Structure, vol. 41, no. 2, pp. 265-279, 1991.

[13] P. Donthireddy and K. Chandrashekhara, "Modeling and shape control of composite beams with embedded piezoelectric actuators," Composite Structures, vol. 35, no. 2, pp. 237244, 1996.

[14] J. H. Han and I. Lee, "Analysis of composite plates with piezoelectric actuators for vibration control using layerwise displacement theory," Composites Part B, vol. 29, no. 5, pp. 621-632, 1998.

[15] C. M. M. Soares, C. A. M. Soares, and V. M. F. Correia, "Optimal design of piezolaminated structures," Composite Structures, vol. 47, no. 1-4, pp. 625-634, 1999.

[16] V. M. F. Correia, M. A. A. Gomes, A. Suleman, C. M. M. Soares, and C. A. M. Soares, "Modelling and design of adaptive composite structures," Computer Methods in Applied Mechanics and Engineering, vol. 185, no. 2-4, pp. 325-346, 2000.

[17] M. I. Frecker, "Recent advances in optimization of smart structures and actuators," Journal of Intelligent Material Systems and Structures, vol. 14, no. 4-5, pp. 207-216, 2003.

[18] Y. J. Yan and L. H. Yam, "Mechanical interaction issues in piezoelectric composite structures," Composite Structures, vol. 59, no. 1, pp. 61-65, 2003.

[19] A. Carlos, S. Mota, M. Cristovao, S. Mota, and V. M. F. Correia, "Modeling and design of laminatedcomposite structures with integrated sensors and actuators," Computational Mechanics for the Twenty-First Century, pp. 165-185, 2000.

[20] C. Chee, L. Tong, and G. P. Steven, "Piezoelectric actuator orientation optimization for static shape control of composite plates," Composite Structures, vol. 55, no. 2, pp. 169-184, 2002.

[21] A. Zabihollah, R. Ganesan, and R. Sedaghati, "Sensitivity analysis and design optimization of smart laminated beams using layerwise theory," Smart Materials and Structures, vol. 15, no. 6, pp. 1775-1784, 2006.

[22] J. Arora, Introduction to Optimization Design, McGraw-Hill, New York, NY, USA, 1989. 

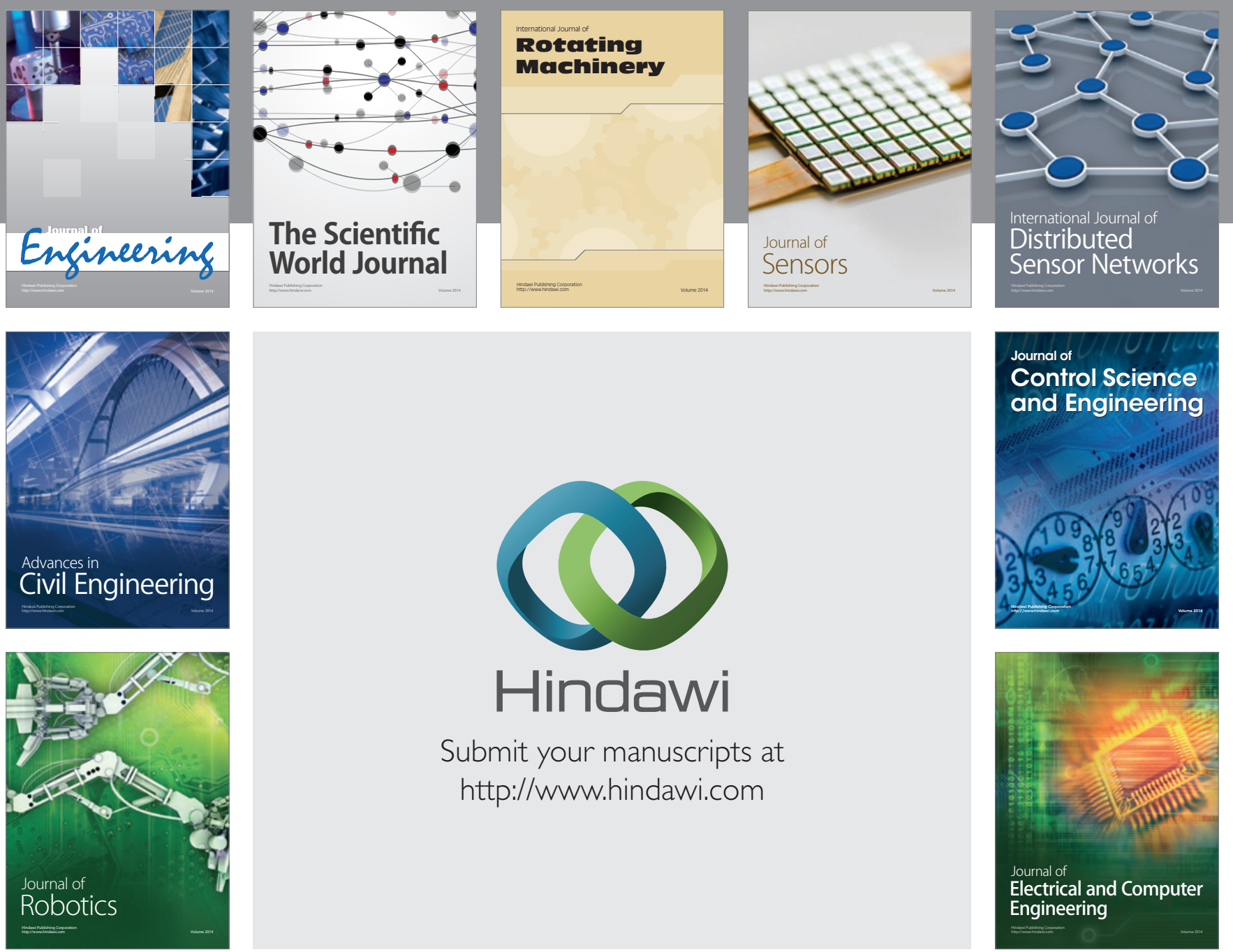

Submit your manuscripts at

http://www.hindawi.com
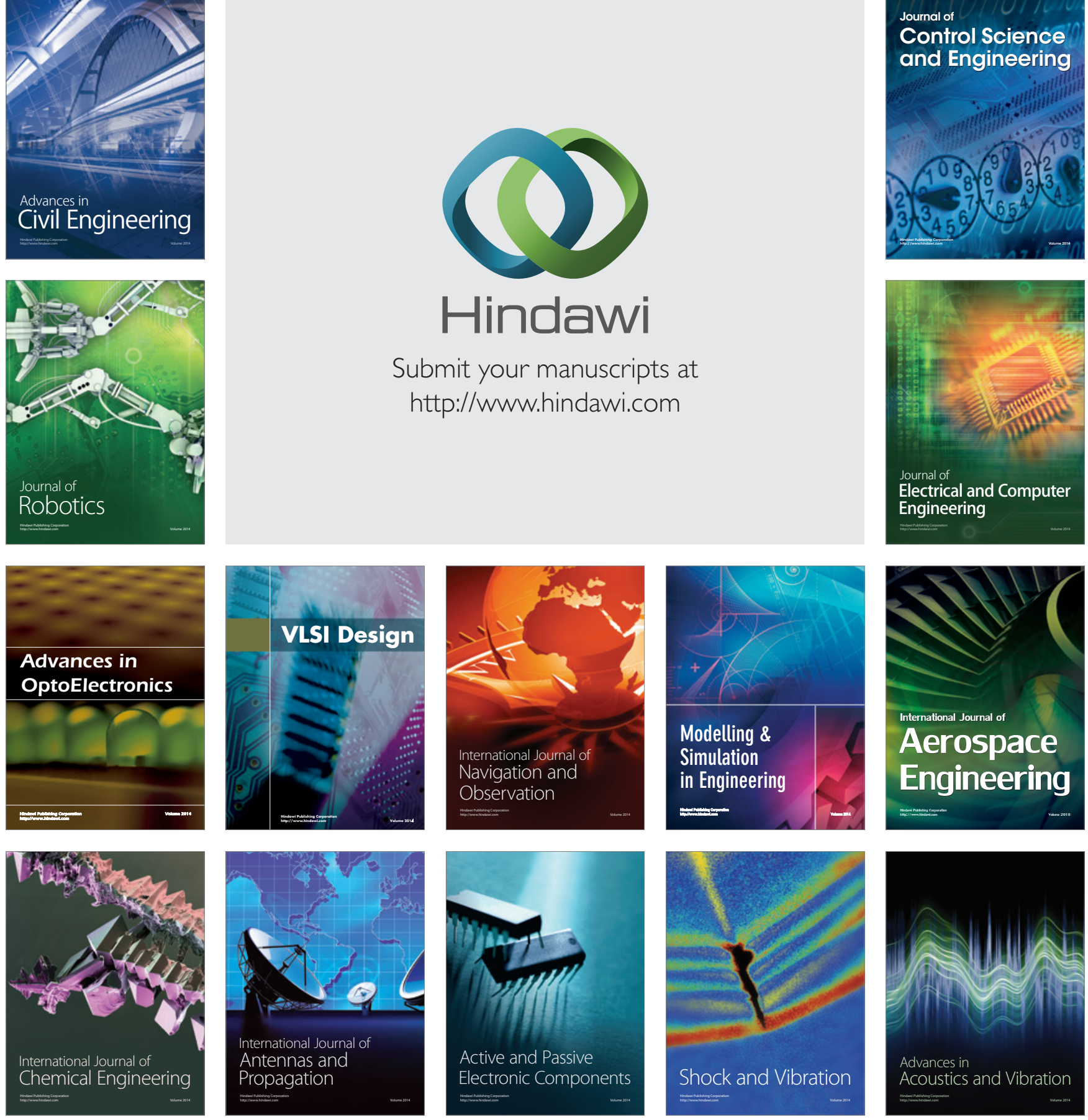